\title{
Relaciones familiares, prácticas educativas de los padres y valores de los adolescentes angoleños
}

\author{
Melchor Gutiérrez ${ }^{1 *}$, Ana María Santos ${ }^{2}$, Patricia Sancho ${ }^{1}$ y Laura Galiana ${ }^{1}$ \\ ${ }^{1}$ Facultad de Psicología, Universidad de Valencia (España) \\ ${ }^{2}$ Instituto Nacional de Investigação, Desenvolvimento e Ensino (Luanda, Angola)
}

(Recibido el 3 de Mayo 2012; Aceptado el 16 de Octubre 2012)

RESUMEN. El objetivo de este trabajo ha sido triple. Primero, analizar las propiedades psicométricas los instrumentos utilizados para evaluar la percepción de las Relaciones familiares, la Socialización familiar y los Valores de los adolescentes angoleños. Segundo, predecir los Valores de los adolescentes a partir de las Relaciones familiares y la Socialización familiar. Tercero, analizar las diferencias de valores de los adolescentes según el sexo. Para ello, 917 adolescentes (384 varones, 533 mujeres) de 14 a 17 años $(M=15.68 ; D T=1.06)$ completaron versiones portuguesas de los instrumentos antes señalados. Una vez analizadas las propiedades psicométricas de los instrumentos, se desarrolló un Modelo de Ecuaciones Estructurales (SEM) controlando por sexo, con las Relaciones familiares y la Socialización familiar como variables independientes y los Valores de los adolescentes como variables dependientes. Los resultados han mostrado aceptables propiedades psicométricas de las versiones portuguesas de los instrumentos. En cuanto al modelo explicativo de los Valores, la Funcionalidad familiar predice los valores Logro, Universalismo, Seguridad y Conformidad; el Apoyo predice los valores Benevolencia y Conformidad; las Dificultades familiares se relacionan negativamente con Logro y Conformidad; y el Castigo se relaciona negativamente con Logro, Benevolencia y Conformidad. La variable sexo apenas ha resultado diferenciadora en los valores de los adolescentes. Los chicos priorizan el Universalismo y las chicas la Conformidad. Se discuten los resultados con vistas a su aplicación práctica para la socialización de los adolescentes. Palabras clave: disciplina familiar, socialización familiar, Angola, internalización de valores.

Family relations, parents' educational practices, and Angolan adolescents'values

ABSTRACT. The purpose of this study was three-fold. First, to analyze psychometric properties of the instruments used to evaluate the perceived Family relations, Familiar socialization and Angolan adolescents' Values. Second, to predict the adolescents' Values based on Family relations and Family socialization. Third, to analyze adolescents' Values sex differences. For this aim, 917 adolescents (384 males, 533 females) ages 14 to 17 years $(M=15.68 ; S D=1.06)$ completed Portuguese translations of the Family relations, Family socialization and Value questionnaires. When psychometric properties of the instruments were 
assessed, a Structural Equation Model (SEM) controlling for sex was carried out, with Family relations and Family socialization as independent variables, and adolescents' Values as dependent variables. Results showed acceptable psychometric properties of the Portuguese versions of the instruments. Related to the SEM, Familiar functioning predicted Achievement, Universalism, Security and Conformity; Parents' Support predicted Benevolence and Conformity; Family difficulties were negatively related with Achievement and Conformity; and Punishment/coercion were negatively related with Achievement, Benevolence and Conformity. The sex variable only differentiated two adolescents' values. Boys assigned priority to Universalism and girls assigned priority to Conformity. These findings are discussed with regard to the implications to adolescents' socialization.

Keywords: family discipline, family socialization, Angola, values internalization.

\section{INTRODUCCIÓN}

\section{Contextualización del objeto de estudio}

Angola comenzó a vivir un contexto de paz apenas en 2002. Antes, pasó por sucesivas guerras que duraron décadas y dejaron serias consecuencias en la sociedad y migración forzosa y en masa de gran parte de la población hacia Luanda, la capital. En este contexto se encuentra una gran parte de los adolescentes angoleños, estimados en un $60 \%$ del total de la población del país. Éstos, sufrieron directa o indirectamente los conflictos armados y los trabajos forzados (Wessells y Monteiro, 2006).

\section{Fundamentación teórica}

La socialización es el proceso por el cual los niños adquieren las habilidades sociales, emocionales y cognitivas necesarias para funcionar en la comunidad social (Bugental y Grusec, 2006). Aunque la socialización tiene lugar en diversos contextos, son los padres los primeros agentes de socialización de los niños y adolescentes, es decir, el primer contexto es la familia. La socialización es un proceso bidireccional, un complejo proceso de interacciones entre los hijos y la conducta de los padres (Collins, Maccoby, Steinberg, Hetherington y Bornstein, 2000).

El uso que hacen los padres de técnicas específicas de disciplina, también denominadas técnicas educativas, de crianza o de socialización, y el tipo de interacciones de los padres con sus hijos, afectan diferencialmente a la internalización de los valores de estos últimos. Tales técnicas también afectan a otros aspectos de su socialización, es decir al proceso de convertirse en personas competentes y con habilidades socioemocionales y criterio personal que les permitan guiar sus propias acciones y contribuir a la comunidad social (Grusec y Goodnow, 1994; Grusec, Goodnow y Kuczynski, 2000). 
Gran parte de la investigación se ha centrado en la disciplina y su papel en el control de la conducta de los hijos. Este interés por la disciplina ha continuado hasta el momento actual, tratando de comparar la efectividad de diferentes técnicas de disciplina empleadas por los padres sobre diversas formas de desarrollo social de los hijos (Grusec y Davidov, 2010). Una cuestión a destacar es que una disciplina efectiva implica la minimización de la percepción de los hijos en cuanto a control externo, lo cual promueve sus sentimientos de autodirección, elemento importante de la autonomía (Grolnick, Deci y Ryan, 1997). La cantidad de control que se debe administrar ha de ser suficiente para producir la conducta deseada pero, al mismo tiempo, no ser tan fuerte como para amenazar la autonomía, lo cual socavaría la internalización. No obstante, un control firme pero sensible por parte de los padres ha mostrado mejores resultados sobre la socialización de los hijos que un control estricto y duro, y mejor que un control laxo o la ausencia de control (Barber, 1996).

La minimización del poder coercitivo es necesaria porque una importante meta de la socialización en el dominio del control es que los niños internalicen los valores, esto es, que consideren los valores de los padres y de la sociedad como suyos propios sin necesidad de que la presión social les fuerce a ello. La clave de la internalización de los valores es la percepción de que los principios guía de comportamiento han sido autogenerados (Bugental y Grusec, 2006). La conducta socialmente aceptable debe estar motivada no por anticipación ni consecuencias externas sino por factores intrínsecos o internos (Grusec y Goodnow, 1994).

Una reciente tendencia en el estudio de las prácticas parentales ha surgido de refinar la clasificación tripartita de las relaciones padres-hijos inicialmente popularizada por Schaefer (1965): aceptación/rechazo, control psicológico/autonomía psicológica y control firme/control laxo (Barber, Maughan y Olsen, 2005). El control conductual parental se refiere a las estrategias que emplean los padres para intentar regular las conductas de los hijos de acuerdo con normas familiares y sociales establecidas. El control psicológico parental se refiere a las estrategias de los padres que no son responsivas a las necesidades emocionales y psicológicas de los niños y que ahogan sus expresiones de independencia y autonomía (Barber, 1996; 2002).

Para analizar las relaciones entre las prácticas educativas de los padres y la socialización de los adolescentes, otros autores han considerado dos constructos ortogonales: exigencia, o grado en que los padres hacen demandas y exigencias a sus hijos, y responsividad, o grado en que los padres responden a las demandas de sus hijos. La combinación de estas dimensiones ha dado lugar a los siguientes cuatro estilos parentales: autorizativo o democrático (se caracteriza por una alta aceptación/implicación y una alta coerción/imposición), autoritario (con alta coerción/imposición y baja implicación/aceptación), indulgente o permisivo (padres con alta implicación/aceptación y baja coerción/imposición), y negligente o indiferente (con baja aceptación/implicación y bajo nivel de coerción/imposición de normas) (Baumrind, 1991; Maccoby y Martin, 1983; Steinberg, Lamborn, 
Darling, Mounts y Dornbusch, 1994). Estudios longitudinales (Baumrind, 1971, 1991) han mostrado una importante asociación entre el buen ajuste de los niños y las prácticas parentales consistentes, firmes, cálidas y apoyativas. Los resultados de investigación indican que las influencias comienzan muy temprano y continúan en la adolescencia, y que el estilo parental no cambia de forma drástica en el transcurso de los años (Fleming, 2005).

Pero como afirman Grusec y Davidov (2010), al considerar la socialización de los hijos no se deben tener en cuenta únicamente las prácticas educativas empleadas por los padres, sino también otras variables del contexto de socialización. Por ello, en este trabajo hemos incluido la percepción que los hijos tienen de las relaciones entre los miembros de su familia. Gimeno, Córdoba, Meléndez y Cerviño (2004) enfatizan la importancia de la percepción que cada miembro de la familia tiene del sistema familiar como una herramienta útil para valorar de forma precisa las relaciones familiares. Estos autores consideran que no sólo es importante conocer la realidad objetiva del funcionamiento familiar, sino también saber cómo la percibe y qué significado le atribuye cada miembro a las diferentes dimensiones de la vida familiar. Se sabe que las familias funcionales son capaces de crear un entorno que facilita el desarrollo personal de sus miembros, y que la funcionalidad aparece asociada a diferentes características del sistema familiar y a la internalización de valores y socialización de los niños y adolescentes en todos sus ámbitos (Caprara, Regalia, Scabini, Barbaranelli y Bandura, 2004).

Schwartz (1992) propuso una tipología universal de valores basada en diez tipos motivacionales (Figura 1): Universalismo (comprensión y protección del bienestar de todos y de la naturaleza), Benevolencia (preocupación por el bienestar de las personas próximas), Conformidad (restricción de acciones e impulsos que pueden violar las expectativas sociales y normas), Tradición (respeto, compromiso y aceptación de las costumbres e ideas de una cultura o religión), Seguridad (armonía y estabilidad de la sociedad, de las relaciones y del "self"), Poder (mantenimiento de una posición social dentro de un sistema social), Realización (búsqueda del éxito personal por medio de demostraciones de competencia de acuerdo con las reglas sociales), Hedonismo (placer y gratificación para sí mismo), y Estimulación (novedad y estimulación en la vida). El modelo teórico de Schwartz también prevé una estructura dinámica entre los tipos motivacionales de forma que las personas tienden a mostrar alta prioridad por los tipos motivacionales mutuamente compatibles, y baja prioridad por los tipos motivacionales conflictivos. A su vez, los diez dominios motivacionales pueden agruparse en cuatro dominios de segundo orden (Schwartz, 2007; Schwartz y Bardi, 2001; Schwartz y Boehnke, 2004): (1) Autobeneficio: agrupa poder, logro y hedonismo; (2) Autotrascendencia: agrupa universalismo y benevolencia; (3) Apertura al Cambio: agrupa hedonismo, estimulación y autodirección; (4) Conservadurismo: incluye seguridad, conformidad y tradición. Los dominios motivacionales de segundo orden representan las relaciones de compatibilidad dentro de cada agrupamiento y también las relaciones de conflicto entre ellos. De esta manera, forman dos dimensiones bipolares: Autobeneficio vs. Autotrascendencia y Aper- 
tura al Cambio vs. Conservadurismo (Fontaine, Poortinga, Delbeke y Schwartz, 2008; Schwartz, 2007). La primera dimensión contrasta la búsqueda de éxito personal con la búsqueda del bienestar de los otros; la segunda dimensión, opone el énfasis en la independencia de acción y pensamiento a la auto-restricción que promueve la preservación de la estabilidad.

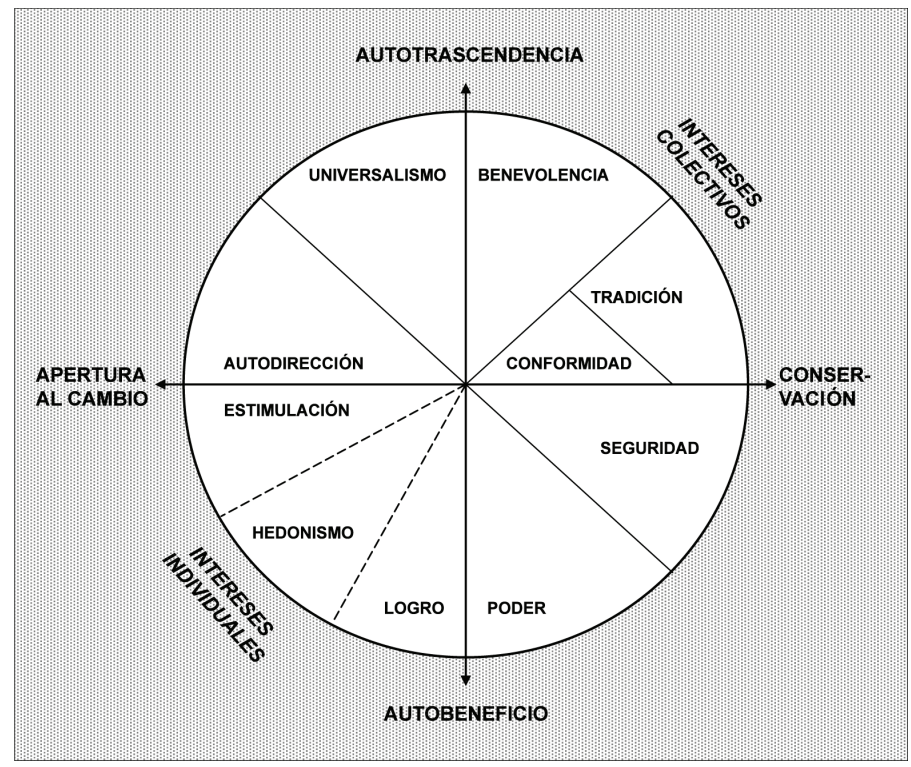

Figura 1. Modelo teórico de Schwartz (1992) de las relaciones entre dominios de valor.

Aunque no abundan las investigaciones sobre los correlatos de valor de los roles sexuales (Schwartz y Rubel, 2009), algunos estudios como el realizado por Prince- Gibson y Schwartz (1998) muestran que los varones priorizan valores instrumentales de poder y logro, mientras que las mujeres se orientan a valores de relación. Schwartz y Rubel (2005) encontraron, con pequeñas diferencias, que los hombres atribuyen de manera consistente mayor importancia que las mujeres a los valores poder, estimulación, hedonismo, logro y autodirección; y lo contrario ocurre con los valores benevolencia y universalismo, y menos consistente para el valor seguridad. No encontraron diferencias por sexo en los valores tradición y conformidad. Por otro lado, Llinares (2003) encontró que los varones adolescentes priorizaron en mayor medida valores de autobeneficio, es decir, valores hedonistas, de poder, de estimulación y de logro, mientras que las mujeres priorizaron valores de autotrascendencia, esto es, valores universalistas, de benevolencia y de tradición.

Diversos autores han referido la influencia de modelos de relaciones familiares con los valores de los adolescentes como estructura jerárquica definida (Gru- 
sec y Goodnow, 1994; Grusec et al., 2000; MacDonald, 1997; Padilla-Walker, 2007; Rudy y Grusec, 2001; Tam y Lee, 2010). Sin embargo, salvo algunas excepciones (ej. Aluja, del Barrio y García, 2005; Martínez y García, 2007, 2008), la internalización de los valores de los adolescentes como resultado de las prácticas educativas de los padres, no ha sido analizada.

En concreto, Aluja et al. (2005) relacionaron los tipos de disciplina que habían empleado los padres, con la importancia que les atribuyeron los hijos a los valores individuales y colectivos. Los valores Benevolencia y Seguridad correlacionaron positivamente con Afecto emocional y negativamente con Rechazo. Por otro lado, los adolescentes que valoraron alto el Poder social, también describieron a sus padres como más apoyativos. La Sobreprotección se relacionó positivamente con el Poder social en los chicos, y negativamente con la Seguridad en las chicas. Los chicos valoraron más que las chicas el Poder social, mientras que los valores Benevolencia y Seguridad mostraron el patrón opuesto. Mediante regresión lineal, encontraron que los mejores predictores del valor Benevolencia fueron el Afecto emocional (en positivo) y el Apoyo (en negativo). En cuanto al Poder social, la variable mejor predictora fue el Rechazo, tanto en chicos como en chicas. La Seguridad fue predicha por la Agresividad y el Afecto emocional en los chicos, y la Agresividad y el Rechazo en las chicas.

Martínez y García (2007) estudiaron muestras españolas y relacionaron cuatro estilos educativos parentales (autorizativos, autoritarios, indulgentes y negligentes), con las puntuaciones de los adolescentes en dos variables diferentes: la prioridad que daban a los valores de auto-trascendencia (universalismoy benevolencia) y la que dieron a conservación (seguridad, conformidad y tradición), de acuerdo con el esquema de Schwartz (1992). Los resultados mostraron que los adolescentes procedentes de familias indulgentes alcanzaban las mismas o mayores puntuaciones en internalización de valores de auto-trascendencia y conservación que los adolescentes procedentes de familias autorizativas. Por otro lado, se confirmó la relación entre los padres caracterizados por la aceptación y el compromiso, con una alta internalización de los valores: los adolescentes procedentes de ambientes autorizativos e indulgentes (ambos caracterizados por alta aceptación/compromiso) dieron mayor prioridad a los valores analizados, mientras que los adolescentes con padres autoritarios y negligentes (ambos caracterizados por baja aceptación/compromiso, generalmente asignaron una menor prioridad a estos valores. El sexo de los adolescentes no apareció como variable diferenciadora.

En otro trabajo, Martínez y García (2008) con muestras brasileñas, encontraron que los adolescentes con padres autorizativos dieron mayor prioridad a los valores analizados (universalismo, benevolencia, conformidad, tradición y seguridad), que los adolescentes con familias negligentes y autoritarias, pero no encontraron diferencias entre adolescentes con padres indulgentes y autorizativos en la prioridad dada a los cinco valores antes señalados. Tampoco estos autores encontraron diferencias en función del sexo de los adolescentes.

A pesar de la importancia que supone conocer las relaciones entre el adoles- 
cente y su ambiente familiar, y el aprendizaje de valores que le son subyacentes, no aparece en la literatura especializada información alguna que analice estos campos de forma sistémica en el contexto angoleño. Por ello, con esta investigación hemos pretendido contribuir a mejorar la comprensión de la conexión entre las relaciones familiares y las prácticas educativas parentales con la internalización de valores de los adolescentes en el contexto específico de Angola. Los objetivos han sido: (1) Validar los instrumentos aplicados para evaluar la percepción de las relaciones familiares, las prácticas educativas de los padres y los valores de los adolescentes angoleños. (2) Someter a prueba un modelo estructural explicativo de los valores de los adolescentes a partir de las relaciones familiares y las prácticas educativas de los padres. (3) Analizar las diferencias por sexo en la internalización de valores de los adolescentes.

Hipótesis: (1) Las relaciones familiares positivas se relacionarán positivamente con los valores de los adolescentes, mientras que las dificultades familiares lo harán de forma negativa. (2) Las prácticas educativas de los padres fundamentadas en el apoyo presentarán relaciones positivas con los valores de los adolescentes, mientras que el castigo y el control parental se relacionarán de manera negativa. (3) De acuerdo con la literatura, los adolescentes varones priorizarán valores de autobeneficio y las mujeres priorizarán valores de autotrascendencia.

\section{MÉTODO}

\section{Participantes}

Participaron 917 adolescentes de 14 a 17 años $(M=15.68 ; D T=1.06)$, $41.87 \%$ varones y $58.13 \%$ mujeres, residentes en la provincia de Luanda (Angola), reclutados en 8 escuelas públicas de los municipios de Sambizanga, Rangel y Cazenga. En cuanto a nivel educativo, se encontraban repartidos entre las clases 7 y 12 (que se corresponden aproximadamente con los niveles de $1^{\circ}$ de ESO hasta $2^{\circ}$ de Bachiller del sistema educativo español). El muestreo fue por conveniencia, a través de colaboradores. A pesar de que el muestreo fue incidental, los participantes pueden considerarse en cierto modo representativos de la población angoleña de este tramo de edad puesto que, aunque residen actualmente en torno a Luanda, provienen de diversos lugares del país, tras numerosos desplazamientos forzosos ocasionados por sucesivos conflictos armados (Wessells y Monteiro, 2006).

\section{Instrumentos}

Cuestionario de Relaciones Familiares. Para evaluar las relaciones familiares se utilizó una versión de la Escala de Competencia Familiar (EFC) de Gimeno et al. (2004). Consta de 34 items, cuyos contenidos se refieren a los factores de comunicación y cooperación entre los familiares, dimensiones de tipo relacional que resultan buenos indicadores de la funcionalidad familiar (ej.: "los miembros 
de mi familia expresan con facilidad afecto e interés por los demás”), y las dificultades familiares (ej.: "discutimos mucho en la familia y nunca resolvemos el problema"). Los adolescentes respondieron en una escala tipo Likert desde (1) Totalmente en desacuerdo, hasta (5) Totalmente de acuerdo.

Cuestionario de Socialización Familiar. Para evaluar las prácticas de socialización de los padres, se utilizó el SOC-30, de García et al. (1994), formado por 30 items que evalúan la percepción que los hijos tienen sobre las prácticas educativas utilizadas por los padres. Las dimensiones que evalúa el SOC-30 son: Apoyo: (ej.: "mis padres me demuestran con palabras y gestos que me quieren"); Castigo/coerción (ej.: "mis padres me castigan, incluso por cometer pequeñas faltas"); Sobreprotección/control (ej.: "mis padres me prohíben hacer cosas que otros jóvenes de mi edad suelen hacer, por miedo a que me suceda algo"); Reprobación (ej.: "mis padres me dicen que no están de acuerdo con la forma de comportarme en casa"). Las opciones de respuesta comprendían desde (1) Totalmente en desacuerdo, hasta (5) Totalmente de acuerdo.

Cuestionario de Valores. Para medir la internalización de valores de los adolescentes se utilizó una versión española (Molpeceres, 1997) del Cuestionario de Valores de Schwartz (1992). Este autor considera el sistema de valores compuesto por diez factores: Poder (ej.: "poder social, control sobre los demás"), Logro (ej.: "fortuna, posesiones materiales"), Hedonismo (ej.: "placer, satisfacción de deseos"), Estimulación (ej.: "una vida variada, llena de desafíos"), Autodirección (ej.: “independencia, autosuficiente), Universalismo (ej.: "unión con la naturaleza"), Benevolencia (ej.: "abierto, tolerante con las ideas de los demás"), Tradición (ej.: "respeto por la tradición, preservar las costumbres"), Conformidad (ej.: "humildad, tratar de pasar desapercibido"), Seguridad (ej.: "seguridad familiar, seguridad de las personas que quiero"). La escala de respuesta comprendía desde (1) Ninguna importancia en mi vida, hasta (5) Mucha importancia en mi vida.

\section{Procedimiento}

Tras obtener el permiso del Ministerio de Educación de Angola, contactamos con los directores de los centros donde se encontraba escolarizada la muestra, para explicarles la investigación y solicitar su colaboración. Todos los participantes se encontraban oficialmente escolarizados, no obstante, el 90\% de ellos fueron entrevistados en sus colegios y el $10 \%$, por no asistir regularmente a las clases, fueron reclutados aprovechando la realización de actividades deportivas organizadas por diversas ONGs y la Asociación Amiga. Todos los participantes lo hicieron voluntariamente, con el consentimiento de sus padres o cuidadores. Los instrumentos fueron aplicados por un grupo de colaboradores entrenados al efecto. Todos los instrumentos fueron traducidos al portugués siguiendo el procedimiento de doble traducción (español-portugués y viceversa). 


\section{Análisis}

Se analizaron los datos a cuatro niveles. (1) Cálculo de los factores de cada cuestionario, a través de Análisis Factoriales Exploratorios (AFEs), mediante el paquete estadístico SPSS 17. (2) Análisis Factoriales Confirmatorios (AFCs) con el programa EQS 6.1 (Bentler, 2005) para comprobar la adecuación de la estructura factorial de los instrumentos con la muestra estudiada. (3) Cálculo de la consistencia interna de cada factor, a partir del coeficiente alfa de Cronbach. (4) Modelos de Ecuaciones Estructurales (SEM) con variables observables (path análisis) para predecir los valores de los adolescentes a partir del funcionamiento familiar y las prácticas educativas de los padres.

Los modelos confirmatorios y los path análisis se han estimado mediante máxima verosimilitud con correcciones robustas de Satorra-Bentler en los errores estándar y estadísticos e índices de ajuste (Finney y DiStefano, 2006), dada la no normalidad de las variables. Para evaluar el ajuste de los modelos propuestos se han utilizado los índices que habitualmente recomienda la literatura especializada. Se ha calculado el CFI y GFI para los que suele considerarse el valor .90 o superior como apropiado para aceptar el modelo; el SRMR y el RMSEA, medidas de cuantía del error, con valores menores de .08 y .06 (respectivamente) considerados aceptables para afirmar que un modelo es plausible; y la prueba chi-cuadrado (Kaplan, 2000). Para aceptar el ajuste de un modelo, no debe considerarse un sólo índice, sino el resultado de varios en su conjunto, por ejemplo el CFI, SRMR y el RMSEA, así como otras combinaciones posibles (Hu y Bentler, 1999).

\section{RESULTADOS}

\section{Propiedades psicométricas de los instrumentos}

Teniendo en cuenta que los instrumentos utilizados no contaban con versiones validadas en el contexto de Angola, se procedió, primeramente, a extraer los componentes principales de cada cuestionario, mediante Análisis Factoriales Exploratorios (AFEs), y posteriormente se sometieron los modelos obtenidos a los correspondientes Análisis Factoriales Confirmatorios (AFCs). Para ello, dado que el tamaño de la muestra total lo permitía, se dividió en dos mitades (50\% al azar) y se utilizó una de ellas como "muestra de desarrollo" para los AFEs y la otra como "muestra de validación” para los AFCs.

Cuestionario de Relaciones Familiares. El AFE desarrollado con este cuestionario proporcionó dos dimensiones: Funcionalidad familiar y Dificultades familiares. El AFC al que se sometió este modelo, aportó aceptables índices de ajuste a los datos $\left(\chi_{208}^{2}=520.092, \mathrm{p}<.001, \mathrm{CFI}=.902, \mathrm{GFI}=.892, \mathrm{SRMR}=\right.$ .072 , RMSEA $=.057)$, ratificando las dos dimensiones anteriormente señaladas. Los índices de consistencia interna obtenidos fueron: Funcionalidad familiar con $\alpha=.751$, y Dificultades familiares con $\alpha=.709$ (ver Tabla 1). 
Tabla 1. Media, Desviación Típica, Fiabilidad y Relaciones Estadísticamente Significativas de las Variables Independientes y las Variables Dependientes entre sí, dentro de la Estructura del Modelo Puesto a Prueba

\begin{tabular}{lcccccccccc}
\hline Variables & 1 & 2 & 3 & 4 & 5 & 6 & 7 & 8 & 9 & 10 \\
\hline 1. Funciona. familiar & & & & & & & & & & \\
2. Dificult.familiares & -.289 & & & & & & & & & \\
3. Castigo/coerción & & .265 & & & & & & & & \\
4. Apoyo & .245 & -.129 & -.160 & & & & & & & \\
5. Sobreprot./control & .109 & -.092 & .182 & .218 & & & & & & \\
6. Logro/poder & & & & & & & & & & \\
7. Universal/autodir. & & & & & & .283 & & & & \\
8. Seguridad/tradic. & & & & & & .252 & .273 & & & \\
9. Benevolencia & & & & & & .338 & .402 & .321 & & \\
10. Conformidad & & & & & & .348 & .280 & .257 & .287 & \\
Media & 3.85 & 2.57 & 2.56 & 3.77 & 3.85 & 3.99 & 3.77 & 3.80 & 4.09 & 4.03 \\
Desviación típica & 0.62 & 0.75 & 0.90 & 0.66 & 0.70 & 0.57 & 0.60 & 0.55 & 0.60 & 0.64 \\
Alfa & .751 & .709 & .765 & .710 & .682 & .710 & .702 & .704 & .691 & .674 \\
\hline
\end{tabular}

Cuestionario de Socialización Familiar. El AFE de este cuestionario aportó tres factores: Castigo/coerción, Apoyo y Sobreprotección/control. Sometido este modelo a un AFC, los índices de ajuste a los datos fueron los siguientes: $\chi_{206}^{2}=$ $498.75, \mathrm{p}<.001, \mathrm{CFI}=.90, \mathrm{GFI}=.88, \mathrm{SRMR}=.07$, RMSEA $=.05$, por lo que puede aceptarse el modelo probado. Los consistencia interna de cada uno de los factores fue: Castigo/coerción $(\alpha=.765)$, Apoyo $(\alpha=.710)$, y Sobreprotección/ control $(\alpha=.682)$.

Cuestionario de Valores. El AFE del Cuestionario de Valores mostró cinco dimensiones: Logro/poder, Universalismo/autodirección, Seguridad/tradición, Benevolencia, y Conformidad. El AFC desarrollado con este modelo de cinco factores aportó unos razonables índices de ajuste: $\chi_{892}^{2}=1726.23, \mathrm{p}<.001, \mathrm{CFI}=$ $.91, \mathrm{GFI}=.87, \mathrm{SRMR}=.06, \mathrm{RMSEA}=.04$, aceptándose la estructura factorial exploratoria probada. Los índices de consistencia interna de los valores fueron: Logro/poder $(\alpha=.710)$, Universalismo/autodirección $(\alpha=.702)$, Seguridad/tradición $(\alpha=.704)$, Benevolencia $(\alpha=.691)$, y Conformidad $(\alpha=.674)$.

\section{Predicción de los valores de los adolescentes}

Se propuso un modelo teórico para predecir los valores de los adolescentes a partir de las relaciones familiares y las técnicas de socialización o prácticas educativas de los padres, controlando por sexo de los adolescentes (Figura 2). 


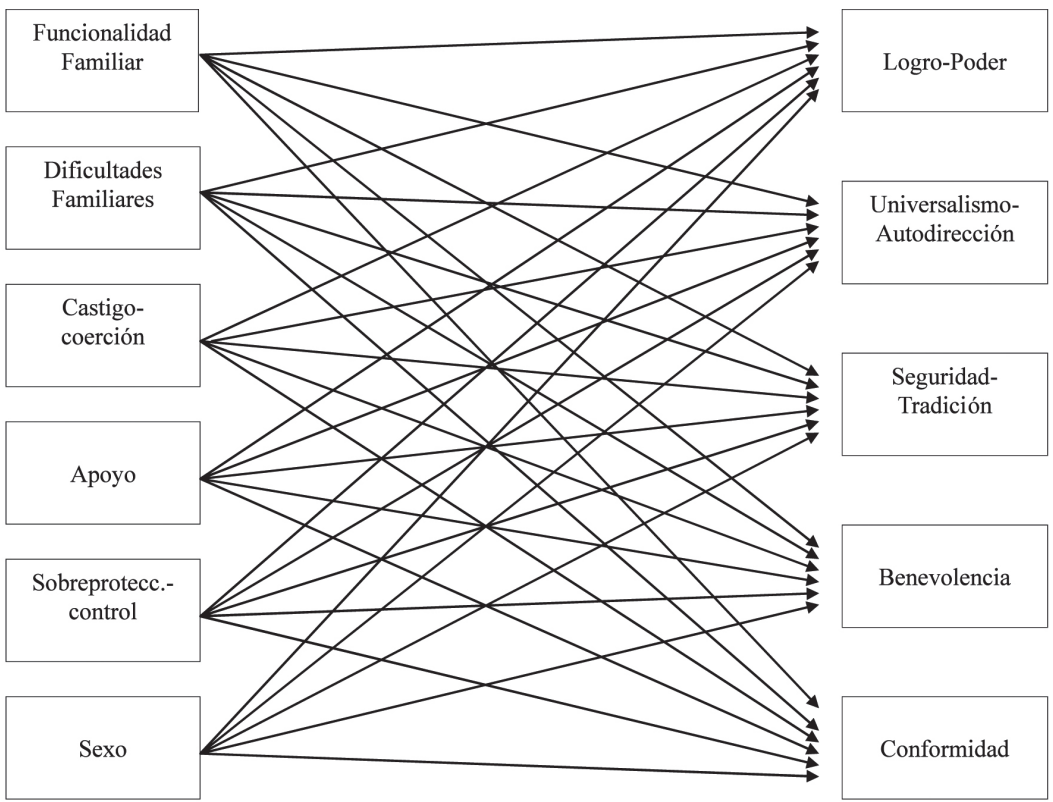

Figura 2. Modelo hipotético saturado para predecir los valores de los adolescentes a partir de las relaciones familiares y las prácticas educativas de los padres.

El modelo inicial es un modelo "saturado" con todas las relaciones posibles, como si se tratara de regresiones múltiples, incluidas las relaciones entre predictores y entre variables dependientes. Este modelo permitió eliminar las relaciones no significativas, tras lo cual se obtuvo el resultado que muestra la Figura 3, con satisfactorios índices de ajuste a los datos: $\chi_{23}^{2}=34.365, \mathrm{p}=.060, \mathrm{CFI}=.989$, $\mathrm{GFI}=.993, \mathrm{SRMR}=.027, \mathrm{RMSEA}=.023$.

Los resultados de este modelo mostraron que la Funcionalidad familiar se relaciona positivamente con los valores Logro/poder, Universalismo/autodirección, Seguridad/tradición y Conformidad, mientras que las Dificultades familiares presentan relaciones negativas con los valores Logro/poder y Conformidad. En cuanto a las técnicas educativas que emplean los padres, el Castigo/coerción se encuentra negativamente relacionado con los valores Logro/poder, Benevolencia y Conformidad, mientras que el Apoyo se relaciona de manera positiva con los valores Benevolencia y Conformidad. La Sobreprotección/control de los padres no presenta relaciones significativas con ninguno de los valores de los adolescentes. Finalmente, el sexo de los adolescentes únicamente presenta relaciones estadísticamente significativas con dos valores, de tal modo que los varones predicen el valor Universalismo/autodirección y las mujeres el valor Conformidad.

Tal como muestra la Figura 3, las variables predictoras de este modelo son capaces de explicar un $7.7 \%$ del valor Logro/poder, un $1.0 \%$ del valor Univer- 
salismo/autodirección, un 1.0\% del valor Seguridad/tradición, un 2.9\% del valor Benevolencia, y un $6.5 \%$ del valor Conformidad. Las variables con mayor capacidad predictiva sobre los valores de los adolescentes han sido, la Funcionalidad familiar $(\beta=.096, .102)$, las Dificultades familiares $(\beta=-.130, .144)$, el Castigo/ coerción $(\beta=-.176,-.129,-.103)$, y el Apoyo de los padres $(\beta=.093)$.

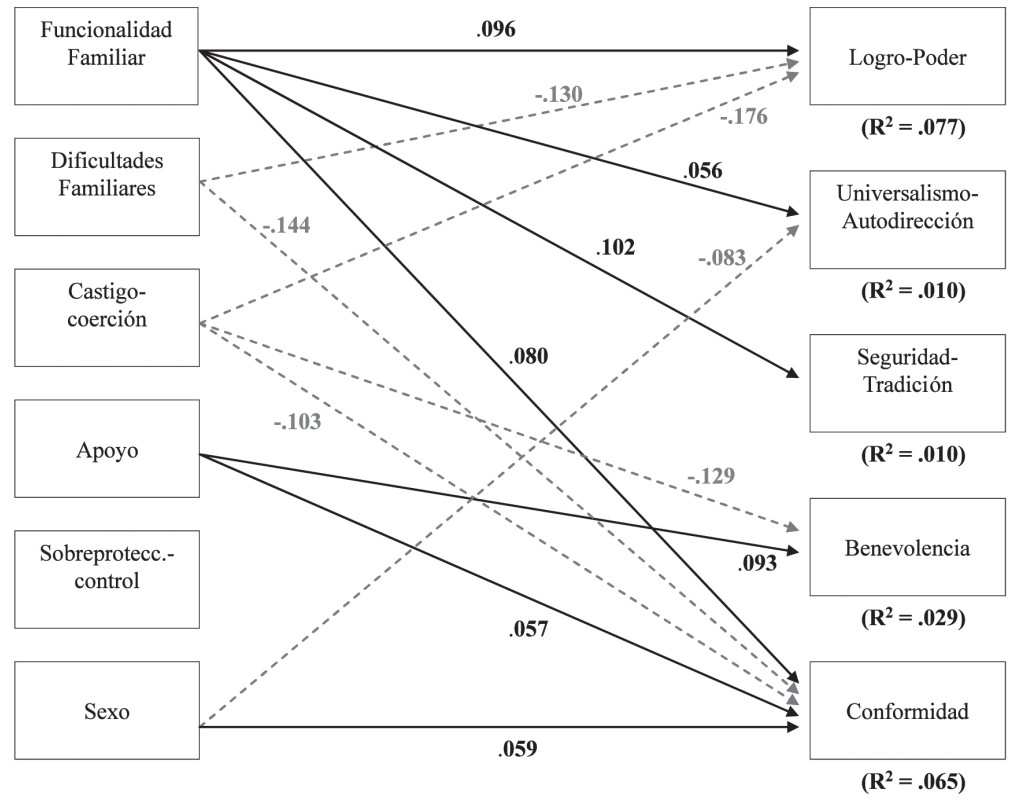

Figura 3. Solución estandarizada para el modelo propuesto de predicción de los valores de los adolescentes a partir de las relaciones familiares y las prácticas educativas de los padres. Nota: Las líneas continuas indican relaciones positivas y las discontinuas relaciones negativas. Todas las relaciones estructurales son estadísticamente significativas $(\mathrm{p}<.01)$. Por razones de claridad expositiva, las correlaciones entre las variables exógenas y los errores se muestran en la Tabla 1.

\section{DISCUSIÓN}

El objetivo de este trabajo ha sido triple: (1) Validar los instrumentos empleados para analizar la percepción de las relaciones familiares, las prácticas educativas de los padres y los valores de los adolescentes angoleños; (2) predecir los valores de los adolescentes a partir de las relaciones familiares y las prácticas educativas de los padres; (3) analizar las diferencias en la internalización de valores de los adolescentes en función del sexo.

Las versiones portuguesas de los cuestionarios de Relaciones familiares y Socialización familiar han mostrado aceptables propiedades psicométricas, 
próximas a las obtenidas por Gimeno et al. (2004) y García et al. (1994), respectivamente. Sin embargo, el cuestionario de Valores ha aportado un resultado factorial ligeramente diferente al propuesto por sus autores, fundiéndose en uno factores como Logro y Poder, Universalismo y Autodirección, o Seguridad y Tradición, valores compatibles en el modelo de Schwart (1992). Estos resultados contrastan también con los de Schwartz y Bardi (2001) o Schwartz y Boehnke (2004), que refieren una importante similitud en los dominios motivacionales de valores en diferentes culturas. Esta menor delimitación de los valores obtenidos en nuestra muestra podría explicarse por razones culturales y educativas y por las características de la sociedad angoleña en el momento actual, aún no suficientemente normalizada tras tantos años de sucesivas guerras.

Con respecto a la primera hipótesis, según la cual las relaciones familiares positivas se relacionarían positivamente con los valores de los adolescentes, y las dificultades familiares lo harían negativamente, los resultados se han mostrado en este sentido, aunque con mayor capacidad predictiva la funcionalidad familiar que las dificultades familiares.

La segunda hipótesis planteaba que las prácticas educativas de los padres basadas en el Apoyo presentarían relaciones positivas con los valores de los adolescentes, y que el Castigo y el Control parental se relacionarían de manera negativa. A la vista de los resultados, la hipótesis se ve cumplida en parte, puesto que el Apoyo parental predice los valores Benevolencia y Conformidad y el poder coercitivo de los padres predice los valores Logro/poder, Benevolencia y Conformidad, en sentido negativo. La Sobreprotección y control parental, sin embargo, no muestra ningún tipo de relación con los valores de los adolescentes. Estos resultados se encuentran en la línea de los obtenidos por Martínez y García $(2007,2008)$ al relacionar los estilos parentales con los valores autotrascendencia y conservación de los adolescentes.

En cuanto a la tercera hipótesis, según la cual los adolescentes varones priorizarían valores de autobeneficio y las mujeres priorizarían valores de autotrascendencia, no se ha visto cumplida, puesto que únicamente hemos encontrado dos relaciones entre sexo y valores, y con niveles de significación bastante bajos. Estos resultados, en contra de lo hipotetizado, coinciden en gran medida con los obtenidos por Martínez y García $(2007,2008)$ con muestras españolas y brasileñas, que no encontraron diferencias en función del sexo de los adolescentes, y se alejan de los obtenidos por Prince- Gibson y Schwartz (1998), Llinares (2003), Aluja et al. (2005) y Schwartz y Rubel (2005), que sí encontraron diferencias entre los valores de los adolescentes varones y los de las mujeres.

Como conclusiones cabe destacar, en primer lugar, que la percepción de las relaciones familiares se muestra con tanto peso como las prácticas educativas de los padres a la hora de predecir los valores de los adolescentes. Cabe pensar que esto sea así porque las prácticas educativas parentales influyen sobre la percepción que los adolescentes tienen de las relaciones familiares, aunque también es posible que las relaciones familiares determinen o influyan sobre las técnicas educativas parentales. En segundo lugar, se refuerza la importancia de 
las prácticas educativas parentales basadas en el afecto, variable propia del estilo autorizativo y democrático, sobre la internalización de valores de los adolescentes. En tercer lugar, cabe resaltar la importancia que este estudio tiene, aunque de momento sea exploratorio, por ser el primero desarrollado en el contexto de Angola, pudiendo servir de base para conducir otros trabajos como los que se sugieren en el apartado siguiente. Finalmente, dada la rapidez con que la sociedad angoleña está cambiando, se hace evidente la necesidad de seguir desarrollando investigaciones sobre estos constructos en el contexto de Angola, para conocer en mayor profundidad el proceso de recuperación social y familiar y poder intervenir en el sentido más conveniente.

\section{Limitaciones y propuestas de investigación.}

Como todo estudio, éste presenta sus limitaciones. Aunque el tamaño de la muestra es aceptable, los datos fueron obtenidos a través de colaboradores (muestra por conveniencia). Futuros estudios deberían contemplar muestras representativas, lo cual será cada vez más factible a medida que Angola vaya organizando el censo de su población. Además, los datos utilizados en este estudio son transversales, por lo que no permiten establecerse relaciones causa-efecto. Por otro lado, en este trabajo hemos analizado únicamente las prácticas educativas de los padres. En futuros trabajos podrían contemplarse los estilos educativos parentales, clasificándolos a partir de los modelos que ofrece la literatura especializada. También podría recogerse el punto de vista de los padres, para contrastarlo con las percepciones que aportan los hijos. Una cuestión a destacar es la conveniencia de contemplar el tipo y calidad de las relaciones padres-hijos en función de características de la muestra como edad y sexo de los jóvenes, clase social, estructura familiar, etnia y afiliación religiosa, tal como sugieren Barber et al. (2005). Una vez superados los primeros estudios exploratorios, siempre necesarios pero insuficientemente explicativos, se podría combinar la metodología cuantitativa con la cualitativa, para una mejor comprensión de la realidad de Angola, dada la gran diversidad de ambientes familiares existentes actualmente. Finalmente, es importante señalar que el modelo sometido a comprobación mediante ecuaciones estructurales es uno de los posibles, pudiendo existir otros modelos capaces de contribuir con nuevas explicaciones a la predicción de los valores de los adolescentes angoleños.

\section{REFERENCIAS}

Aluja, A., del Barrio, V. y García, L. F. (2005). Relationships between adolescents' memory of parental rearing styles, social values and socialisation behavior traits. Personality and Individual Differences, 39, 903-912.

Barber, B. K. (1996). Parental psychological control: Revisiting a neglected construct. Child Development, 67, 3296-3319.

Barber, B. K. (ed.) (2002). Intrusive parenting: How psychological control 
affects children and adolescents. Washington, D. C.: APA Press.

Barber, B. K., Maughan, S. L. y Olsen, J. A. (2005). Patterns of parenting across adolescence. New Directions for Child and Adolescent Development, 108, 5-16.

Baumrind, D. (1971). Current patterns of parental authority. Developmental Psychology Monographs, 4, 99-102.

Baumrind, D. (1991). The influence of parenting style on adolescent competence and substance use. The Journal of Early Adolescence, 11, 56-95.

Bentler, P. M. (2005). EQS program manual. Encino, CA: Multivariate Software, Inc.

Bugental, D. B. y Grusec, J. E. (2006). Socialization processes. En W. Damon y N. Eisenberg (Eds.), Handbook of child psychology: Vol. 3. Social, emotional, and personality development (6th ed., pp. 366-428). New York: Wiley.

Caprara, G. V., Regalia, C., Scabini, E., Barbaranelli, C. y Bandura, A. (2004), Assessment of filial, parental, marital, and collective family efficacy beliefs. $E u$ ropean Journal of Psychological Assessment, 20, 247-261.

Collins, W. A., Maccoby, E. E., Steinberg, L., Hetherington, E. M. y Bornstein, M. H. (2000). Contemporary research on parenting: The case for nature and nurture. American Psychologist, 55, 218-232.

Finney, S. J. y DiStefano, C. (2006). Non-normal and categorical data in SEM. En G.R. Hancock y R.O. Mueller (Eds.), Structural Equation Modeling: A second course (pág. 269-314). Greenwich, CO: Information Age Publishing.

Fleming, M. (2005). Adolescent autonomy: Desire, achievement and disobeying pares between early and late adolescence. Australian Journal of Education and Developmental Psychology, 5, 1-16.

Fontaine, J. R., Poortinga, Y. H., Delbeke, L. y Schwartz, S. H. (2008). Structural equivalence of the values domain across cultures: Distinguishing sampling fluctuations from meaningful variation. Journal of Cross-Cultural Psychology, 39(4), 345-365.

García, F., Molpeceres, M. A., Musitu, G., Allat, P., Fontaine, A. M., Campos, B. y Arango, G. (1994). Evaluación de la socialización familiar. En G. Musitu y P. Allat (Eds.), Psicosociología de la familia (295-320). Valencia: Albatros.

Gimeno, A., Córdoba, I., Meléndez, J. C. y Cerviño, C. (2004). Divergencias en la percepción de la funcionalidad familiar entre padres, madres e hijos adolescentes. Revista Latinoamericano de Psicología, 36(3), 459-470.

Grolnick, W. S., Deci, E. L. y Ryan, R. M. (1997). Internalization within the family: The self-determination theory perspective. En J. E. Grusec y L. Kuczynski (Eds.), Parenting and children's internalization of values: A handbook of contemporary theory (pp. 135-161). New York: Wiley.

Grusec, J. E. y Davidov, M. (2010). Integrating different perspectives on socialization theory and research: A domain-specific approach. Child Development, 81(3), 687-709.

Grusec, J. E. y Goodnow, J. J. (1994). Impact of parental discipline methods on the child's internalization of values: A reconceptualization of current points 
of view. Developmental Psychology, 30(1), 4-19.

Grusec, J. E., Goodnow, J. J. y Kuczynski, L. (2000). New directions in analyses of parenting contributions to children's acquisition of values. Child Development, 71(1), 205-211.

Hu, L. y Bentler, P. M. (1999). Cutoff criteria for fit indices in covariance structure analysis: Conventional criteria versus new alternatives. Structural Equation Modeling, 6(1), 1-55.

Kaplan, D. (2000). Structural equation modeling: Foundations and extensions. Thousand Oaks, CA: Sage.

Llinares, L. I. (2003). Las prioridades de valor en la adolescencia: Diferencias en función del sexo y la edad. Informació Psicològica, 81, 55-62.

Maccoby, E. E. y Martin, J. A. (1983). Socialization in the context of the family: Parent-child interaction. En P. H. Mussen (Ed.), Handbook of child psychology (Vol. 4, pp. 1-101). New York: Wiley.

MacDonald, K. (1997). The coherence of individual development: An evolutionary perspective on children's internalization of parental values. En J. E. Grusec y L. Kuczynski (Eds.), Parenting and children's internalization of values: A handbook of contemporary theory (pp. 362-397). New York: Wiley.

Martínez, I. y García, J. F. (2007). Impact of parenting styles on adolescents' self-esteem and internalization of values in Spain. The Spanish Journal of Psychology, 10(2), 338-348.

Martínez, I. y García, J. F. (2008). Internalization of values and self-esteem among Brazilian teenagers from authoritative, indulgent, authoritarian, and neglectful homes. Adolescence, 43(169), 13-29.

Molpeceres, M. A. (1997). El sistema de valores. Tesis Doctoral no publicada. Universidad de Valencia.

Padilla-Walker, L. M. (2007). Characteristics of mother-child interactions related to adolescents' positive values and behaviors. Journal of Marriage and the Family, 69, 675-686.

Prince-Gibson, E. y Schwartz, S. H. (1998). Value priorities and gender. Social Psychology Quarterly, 61(1), 49-67.

Rudy, D. y Grusec, J. E. (2001). Correlates of authoritarian parenting in individualist and collectivist cultures and implications for understanding the transmission of values. Journal of Cross-Cultural Psychology, 32, 202-212.

Schaefer, E. W. (1965). Children's reports of parental behavior: An inventory. Child Development, 36, 413-424.

Schwartz, S. H. (1992). Universals in the content and structure of values: Theoretical advances and empirical tests in 20 countries. En M. P. Zanna (Ed.), Advances in Experimental Social Psychology (vol. 25, pp. 1-65). NY.; Academic Press.

Schwartz, S. H. (2007). Universalism values and the inclusiveness of our moral universe. Journal of Cross-Cultural Psychology, 38, 711-728.

Schwartz, S. H. y Bardi, A. (2001). Value hierarchies across cultures: Taking a similarities perspective. Journal of Cross-Cultural Psychology, 32(3), 268-290. 
Schwartz, S. H. y Boehnke, K. (2004). Evaluating the structure of human values with confirmatory factor analysis. Journal of Research in Personality, 38, 230-255.

Schwartz, S. H. y Rubel, T. (2005). Sex differences in value priorities: Crosscultural and multimethod studies. Journal of Personality and Social Psychology, 89(6), 1010-1028.

Schwartz, S. H. y Rubel, T. (2009). Cross-national variation in the size of sex differences in values: Effects of gender equality. Journal of Personality and Social Psychology, 97, 171-185.

Steinberg, L., Lamborn, S. D., Darling, N., Mounts, N. S. y Dornbusch, S. M. (1994). Over-time changes in adjustment and competence among adolescents from authoritative, authoritarian, indulgent, and neglectful families. Child Development, 65, 754-770.

Tam, K. y Lee, S. (2010). What values do parents want to socialize in their children? The role of perceived normative values. Journal of Cross-Cultural Psychology, 41(2), 175-191.

Wessells, M. y Monteiro, C. (2006). Psychosocial assistance for youth: Toward reconstruction for peace in Angola. Journal of Social Issues, 62(1), 121-139 